\title{
AMS 3.0: prediction of post-translational modifications
}

\author{
Subhadip Basu ${ }^{\dagger 1}$ and Dariusz Plewczynski*+2
}

\begin{abstract}
Background: We present here the recent update of AMS algorithm for identification of post-translational modification (PTM) sites in proteins based only on sequence information, using artificial neural network (ANN) method. The query protein sequence is dissected into overlapping short sequence segments. Ten different physicochemical features describe each amino acid; therefore nine residues long segment is represented as a point in a 90 dimensional space. The database of sequence segments with confirmed by experiments post-translational modification sites are used for training a set of ANNs.

Results: The efficiency of the classification for each type of modification and the prediction power of the method is estimated here using recall (sensitivity), precision values, the area under receiver operating characteristic (ROC) curves and leave-one-out tests (LOOCV). The significant differences in the performance for differently optimized neural networks are observed, yet the AMS 3.0 tool integrates those heterogeneous classification schemes into the single consensus scheme, and it is able to boost the precision and recall values independent of a PTM type in comparison with the currently available state-of-the art methods.
\end{abstract}

Conclusions: The standalone version of AMS 3.0 presents an efficient way to indentify post-translational modifications for whole proteomes. The training datasets, precompiled binaries for AMS 3.0 tool and the source code are available at http://code.google.com/p/automotifserver under the Apache 2.0 license scheme.

\section{Background}

Post-translational modification (PTM) is the chemical modification of a protein after its translation. During protein synthesis, a protein is build using twenty different amino acids, yet after translation a post-translational modification of amino acids can be observed by attaching to them other biochemical functional groups such as acetate, phosphate, various lipids and carbohydrates, by changing the chemical nature of an amino acid, or by making structural changes, like the formation of disulfide bridges. In the advent of massive (complex and time-consuming) sequencing experiments, the availability of whole proteomes requires accurate computational techniques for investigation of protein modification sites for the high-throughput scale. To address these needs we propose here our improved technique to identify PTM

\footnotetext{
* Correspondence: D.Plewczynski@icm.edu.pl

2 Interdisciplinary Centre for Mathematical and Computational Modeling,

University of Warsaw, 5a Street, 02-106 Warsaw, Poland

+ Contributed equally

Full list of author information is available at the end of the article
}

sites by using artificial neural network, trained on proteins from the current version of Swiss-Prot database [1] and Phospho.ELM dataset [2,3].

The automatic prediction of PTM sites is now very important area of interest for the bioinformatics research community. The currently available PTM prediction tools can be categorized into four major groups based on the used types of classification schemes. The first category includes general PTM related resources like ELM [4] that perform rapid regular expression pattern search in order to predict Eukaryotic Linear Motifs (ELMs) in protein sequences. Another web service, namely PROSITE [5] predicts many types of PTMs based on the consensus of sequence patterns. Consensus based approaches combine several signature recognition methods to scan a given query protein sequence against observed protein signatures. The Scansite tool [6] predicts kinase-specific and signal transduction relevant motifs. The conserved sequence motifs represent footprints of important biochemical properties or biological functions performed by those proteins. GPS approach predicts kinase-specific 
phosphorylation sites from protein primary sequences for 71 different PK groups by family-based phosphorylation scoring technique [7,8]. The PHOSITE [9] algorithm for prediction of phosphorylation sites is based on casebased sequence analysis to obtain predictions with constant specificity and sensitivity.

The second class of methods covers different neural network prediction tools. These include phosphorylation related prediction servers like NetPhos [10] and NetPhosK $[11,12]$, glycosylation based tools like NetOGlyc [13], NetNGlyc, DictyOGlyc [14], YinOYang [15], prediction of cleavage sites in protein sequences [16-18], prediction of $\mathrm{N}$-terminal myristoylation on protein sequences [19] and many others. The most popular among these servers is NetPhosK that allows the user to choice its preferred 'threshold' value during prediction. In our manuscript we present the results with three threshold levels: $0.3,0.5$ and 0.7 for making the predictions and they are called respectively as NetPhosK_0.3, NetPhosK_0.5 and NetPhosK_0.7.

The third category of methods involves several support vector machine (SVM) based prediction techniques. Among the recent works, protein methylation site prediction is attempted using bayesian feature extraction technique and SVM based classifier [20]. In another work, the prediction of Lysine acytelation sites is done using SVM [21] based classifiers. PredPhospho [22] is another SVM based system that attempts to predict phosphorylation sites and the type of kinase that acts at each site. Our previously developed web-server AutoMotifServer (AMS) [23] for prediction of post-translational modification sites in protein sequences, also uses SVM classifier with both linear and polynomial kernels. This software is available freely at http://ams2.bioinfo.pl/. KinasePhos 2.0 [24] is another web server for identifying protein kinase-specific phosphorylation sites based on 9 amino acid long sequences and coupling patterns. There are several options provided in this prediction program, including three different levels of specificity: $90 \%, 95 \%$ and $100 \%$. These three options are named respectively KinasePhos_90, KinasePhos_95 and KinasePhos_100.

The fourth category consists from the remaining other types of machine learning based PTM prediction tools. It includes for example Sulfinator [25] that predicts tyrosine sulfation sites using a combination of HMM models; prediction of glycosylation sites using random forests [26]; PPSP prediction of PK-specific phosphorylation sites [8] that deploys Bayesian decision theory (BDT); and many others. The PPSP predicted the plausible phosphorylation sites accurately for approximately $70 \mathrm{PK}$ (Protein Kinase) groups. For our tests we choose the PPSP_balanced model that seems to provide the best overall performance for all types of protein families. In the important work of Wan et al. [27] the efficient meta predictor is designed that organize and process predictions from individual source prediction algorithms. They compiled and evaluated their technique on four unbiased phosphorylation site datasets, namely the four major protein kinase families: CDK, CK2, PKA and PKC. In addition to the aforementioned classification software/tools, the dbPTM database [28] compiles diverse information on protein post-translational modifications (PTMs), such as the catalytic sites, solvent accessibility of amino acid residues, protein secondary and tertiary structures, protein domains and protein variations. The database includes a majority of the experimentally validated PTM sites from Swiss-Prot, PhosphoELM and O-GLYCBASE. The recent survey [29] describes available resources for predicting kinase-specific phosphorylation sites from sequence properties. They compare strengths and weaknesses of variety of prediction tools, as described above.

Despite almost ten years of work and above reported computational solutions, still we are unable to boost the precision of in silico methods to be really useful in high throughput context of personalized medicine. Therefore, the present research improvements concentrate on two important factors. The first is to further optimize prediction accuracy in comparison with the current state-ofthe-art methods for variety of PTM sites, especially focusing on selecting more informative feature descriptors. The second factor is the speed of a prediction procedure that is needed for the virtual screening of whole proteomes. Therefore, we present here an extensively optimization scheme for selecting the most informative amino acids features, than used for training the very fast machine learning method, namely artificial neural network.

We have improved significantly the accuracy of the previous versions of AMS prediction tool [23,30] using an efficiently designed Multi Layer Perceptron (MLP) pattern classifier. In the current version of the tool, the query protein sequences are dissected into overlapping short segments. Ten different physico-chemical features represent each amino acid from a sequence segment; therefore the nine amino acids segment is represented as the point in a 90 dimensional abstract space of protein characteristics. The MLP used in this work, special Artificial Neural Network (ANN) algorithm, is developed to replicate learning and generalization abilities of human's behavior with an attempt to model the functions of biological neural networks of the human brain. The MLP architecture is build from a feed-forward layered network of artificial neurons, where each artificial neuron in the MLP computes a sigmoid function of the weighted sum of all its inputs.

The MLP based ANNs (see Figure 1) are observed to be capable of classifying highly complex and nonlinear biological sequence patterns, where correlations between 


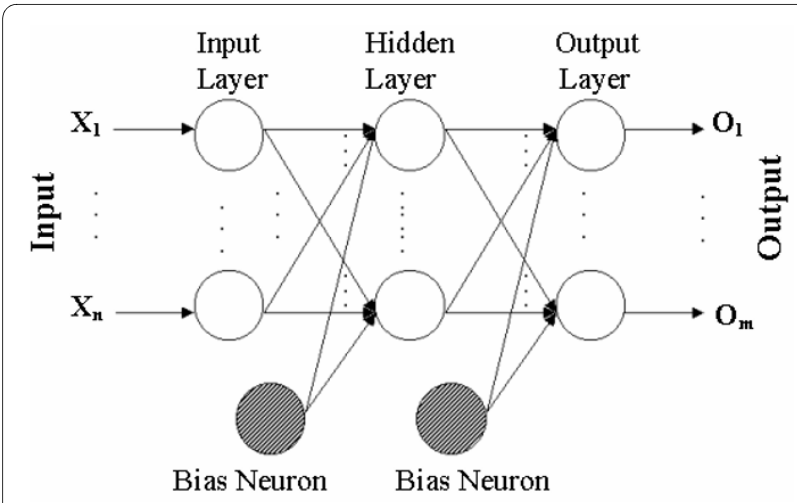

Figure 1 MLP algorithm. A block diagram of an MLP shown as a feed forward layered neural network.

amino acid positions are important. Unlike earlier attempts, in the current design of the neural network we have implemented three different network models for each of the PTM types, by independently optimizing the network weights for three factors: optimum recall (sensitivity); precision; and maximizing the receiver operating characteristics of the prediction model. The consensus build by those three ANNs for each type of PTM gives an additional advantage in comparison to the previously reported ANN based PTM prediction models. In our previous publications on AMS [23,30], the SVM based classification model failed to classify several PTM types with limited number of positive samples. The current MLP based design is much better suited to highly unbalanced ratio between positives and negatives in the training dataset, therefore preferred over the previously chosen SVM based approach.

Summarizing, the AMS 3.0 tool (available at http:// bio.icm.edu.pl/ darman/ams3 and $\underline{\text { http:// }}$ code.google.com/p/automotifserver/) integrates heterogeneous classification schemes for different PTM types, and it is designed to boost both the efficiency and speed in comparison with previously presented computational methods. As discussed earlier, the neural network is designed in much more efficient and unique way to predict post-translational modifications than previously used ANN algorithms. A detailed discussion on the architecture of the neural network, details of the machine learning algorithm is given in the first section. The next section presents the results on several benchmarking datasets, and finally the discussion part of the paper report the major advances of our work.

\section{Results}

The performance of the networks is evaluated on the training and test datasets for each of the PTM types. During training of the feed-forward neural network with back-propagation learning algorithm, the learning rate $(\eta)$ and acceleration factors $(\delta)$ are chosen as 0.8 and 0.8 respectively. The classification performance is described by the following measures of accuracy:

$$
\text { Accuracy }=1-\text { Error }=\frac{T P+T N}{T P+F P+T N+F N},
$$

$\operatorname{Sensitivity}(S n)=\operatorname{Recall}(R)=$ TruePositiveRate $(T P R)=\frac{T P}{T P+F N}$,

$$
\operatorname{Pr} \operatorname{ecision}(P)=\frac{T P}{T P+F P}
$$

$$
\text { FalsePositiveRate }=1-\text { Specifity }=\frac{F P}{F P+T N},
$$

where, TP is the number of true positives, FP is the number of false positives, TN is the number of true negatives, and FN is the number of false negatives. The classification error (E) is used to provide an overall error measure, recall $(R)$ corresponds to the percentage of correct predictions, precision $(\mathrm{P})$ measures the percentage of observed positives that are correctly predicted, true positive rate (TPR) is similar to recall or sensitivity measure and false positive rate (FPR) estimates the false alarm rate or fall-out. We also estimate the receiver operating characteristic (ROC) by plotting the fraction of true positives (TPR) vs. the fraction of false positives (FPR). More specifically, the ROC curves are drawn for both training and test datasets in each of the three runs of experiment, i.e., optimal AUC, recall and precision values. This ROC analysis provides a tool to select possibly optimal network models and to discard suboptimal ones independently from the class distribution. The area under the ROC curve (AUC) is also calculated in the current experiment and presented in Table 1. The AUC is equivalent to the probability that a classifier will rank a randomly chosen positive instance higher than a randomly chosen negative one [31].

The detailed performance analysis of the current software using three random sub-sample validations on training and test datasets of different PTM types is given in the Additional file 1. The three sets of results (corresponding to the AUC, Recall and Precision optimizations) for each PTM type corresponds to the training of the network based on the optimized AUC area, recall and precision values for the random test dataset under consideration. Each such network is trained with possible variations of hidden neurons from 2 to 20 (with step size 2). The number of hidden neurons, for which a given net- 
Table 1: Single Amino-Acids Features Selection.

\begin{tabular}{|c|c|c|c|}
\hline $\begin{array}{l}\text { Accession } \\
\text { number }\end{array}$ & Brief feature description & Reference & Selected/Rejected \\
\hline ARGP820101 & Hydrophobicity index & Eur. J. Biochem. 128, 565-575 (1982) & Rejected \\
\hline BIOV880101 & $\begin{array}{l}\text { Information value for } \\
\text { accessibility; average fraction } \\
35 \%\end{array}$ & Protein Engineering 2, 185-191 (1988) & Selected \\
\hline BIOV880102 & $\begin{array}{l}\text { Information value for } \\
\text { accessibility; average fraction } \\
23 \%\end{array}$ & Protein Engineering 2, 185-191 (1988) & Selected \\
\hline BLAS910101 & $\begin{array}{l}\text { Scaled side chain } \\
\text { hydrophobicity values }\end{array}$ & Analytical Biochemistry 193, 72-82 (1991) & Selected \\
\hline BLAS910101 & $\begin{array}{l}\text { Scaled side chain } \\
\text { hydrophobicity values }\end{array}$ & Analytical Biochemistry 193, 72-82 (1991) & Rejected \\
\hline BULH740101 & $\begin{array}{l}\text { Surface tension of amino acid } \\
\text { solutions: A hydrophobicity } \\
\text { scale of the amino acid } \\
\text { residues }\end{array}$ & Arch. Biochem. Biophys. 161, 665-670 (1974) & Rejected \\
\hline FASG760101 & Molecular weight & $\begin{array}{l}\text { Handbook of Biochemistry and Molecular } \\
\text { Biology, 3rd ed., Proteins - Volume 1, CRC } \\
\text { Press, Cleveland (1976) }\end{array}$ & Rejected \\
\hline HOPA770101 & Hydration number & $\begin{array}{l}\text { Intermolecular Interactions and } \\
\text { Biomolecular Organizations, Wiley, New } \\
\text { York (1977) }\end{array}$ & Selected \\
\hline KRIW710101 & $\begin{array}{l}\text { Side chain interaction } \\
\text { parameter }\end{array}$ & Biochim. Biophys. Acta 229, 368-383 (1971) & Selected \\
\hline KRIW790101 & $\begin{array}{l}\text { Side chain interaction } \\
\text { parameter }\end{array}$ & Biochim. Biophys. Acta 576, 204-228 (1979) & Selected \\
\hline KRIW790102 & $\begin{array}{l}\text { Fraction of site occupied by } \\
\text { water }\end{array}$ & Biochim. Biophys. Acta 576, 204-228 (1979) & Selected \\
\hline KRIW790103 & Side chain volume & Biochim. Biophys. Acta 576, 204-228 (1979) & Selected \\
\hline LAWE840101 & $\begin{array}{l}\text { Transfer free energy, CHP/ } \\
\text { water }\end{array}$ & J. Biol. Chem. 259, 2910-2912 (1984) & Selected \\
\hline OOBM850105 & $\begin{array}{l}\text { Optimized side chain } \\
\text { interaction parameter }\end{array}$ & $\begin{array}{l}\text { Bull. Inst. Chem. Res., Kyoto Univ. 63, 82-94 } \\
\text { (1985) }\end{array}$ & Selected \\
\hline WARP780101 & $\begin{array}{l}\text { Average interactions per side } \\
\text { chain atom }\end{array}$ & J. Mol. Biol. 118, 289-304 (1978) (Gly 0.81) & Rejected \\
\hline
\end{tabular}

List of experimentally chosen features used in the current experiment from the AAindex database with the corresponding feature accession number, brief description and the journal reference.

work is observed to give best possible recognition accuracy, is also listed in the detailed experimental datasheet of the Additional file 1. It may be observed that the high recall value (more than $70 \%$ ), reasonable precision (more than $50 \%$ ) and AUC area (more than 0.75 ) is achieved on independent test datasets of most of the PTM types.

The performance of the current technique is observed to be significantly better for most PTM types in comparison with the results of the previous version of AutoMotif Server, i.e., AMS 2.0. In AMS 2.0, classification results using Support Vector Machine on the training datasets for respective PTM types are shown, which improved the earlier recognition performance of the earlier version of the server AMS 1.0 [23]. A relative comparison of the training set results of our technique with the corresponding results of the AMS 2.0 is shown in Table 2. Detailed results related to respective test set accuracies and due to variations in nomenclature, the current version of Swiss Prot dataset sometimes could not be matched precisely with all the PTM types, experimented in AMS2. However, a detailed experiment is also conducted with the old dataset to show the level of improvement for the current methodology. The results in both cases (with old and new datasets) clearly indicate that the developed software outperforms/improves the performance of the previous server. 
Table 2: Improvement of the performance of the AutoMotifServer 3.0.

\begin{tabular}{|c|c|c|c|c|c|c|c|c|c|}
\hline \multirow[b]{2}{*}{ PTM type } & \multirow[b]{2}{*}{ Agent } & \multicolumn{2}{|c|}{ AMS 2.0} & \multicolumn{3}{|c|}{ AMS 3.0} & \multicolumn{3}{|c|}{ AMS 3.0 (New SwissProt dataset) } \\
\hline & & Recall & Precision & Recall & Precision & AUC & Recall & Precision & AUC \\
\hline 3,4-dihydroxyproline & - & 88,89 & 66,67 & 95,45 & 87,5 & 0,962273 & 97,37 & 97,37 & 0,985088 \\
\hline 4-carboxyglutamate & - & 90,56 & 95,93 & 96,27 & 99,65 & 0,980856 & 96,54 & 99,35 & 0,982076 \\
\hline 4-hydroxyproline & - & 65,59 & 85,31 & 86,88 & 83,94 & 0,905902 & 95,03 & 94,14 & 0,969559 \\
\hline 5-hydroxylysine & - & 12,79 & 84,62 & 97,3 & 38,92 & 0,845236 & 90,16 & 95,93 & 0,948272 \\
\hline $\begin{array}{l}\text { Asymmetric } \\
\text { dimethylarginine }\end{array}$ & - & 84,62 & 82,09 & 94,55 & 94,55 & 0,965227 & - & - & - \\
\hline dihydroxyphenylalanine & - & 29,41 & 76,92 & 86,17 & 86,17 & 0,914601 & - & - & - \\
\hline Glycine amide & - & 84,38 & 90 & 97,92 & 97,92 & 0,977679 & 99,3 & 99,3 & 0,984574 \\
\hline Hydroxyproline & - & 28,8 & 85,71 & 89,62 & 94,06 & 0,942113 & 94,62 & 99,19 & 0,972567 \\
\hline Leucine amide & - & 98,97 & 97,96 & 98,1 & 99,36 & 0,989049 & - & - & - \\
\hline Methionine amide & - & 100 & 93,75 & 100 & 93,75 & 0,666667 & - & - & - \\
\hline $\mathrm{N}$-acetylglycine & - & 79,17 & 90,48 & 97,83 & 97,83 & 0,985797 & - & - & - \\
\hline $\mathrm{N}$-acetylmethionine & - & 100 & 95,42 & 98,4 & 98,93 & 0,990593 & - & - & - \\
\hline $\mathrm{N}$-acetylserine & - & 98,24 & 99,64 & 98,22 & 99,4 & 0,990124 & - & - & - \\
\hline $\mathrm{N}$-acetylthreonine & - & 76,47 & 88,64 & 89,8 & 97,78 & 0,94648 & - & - & - \\
\hline N6-acetyllysine & - & 12,76 & 74,32 & 94,3 & 93,23 & 0,953465 & - & - & - \\
\hline N6, N6, N6-trimethyllysine & - & 7,5 & 60 & 85,71 & 97,5 & 0,926065 & - & - & - \\
\hline $\begin{array}{l}\text { Omega-N-methylated } \\
\text { arginine }\end{array}$ & - & 0 & 0 & 78 & 58,21 & 0,82 & - & - & - \\
\hline Phenylalanine amide & - & 98,59 & 93,33 & 99 & 99 & 0,986667 & - & - & - \\
\hline Phospho & PKA & 63,64 & 77,78 & 90,72 & 86,27 & 0,939608 & 97,39 & 95,39 & 0,983802 \\
\hline Phospho & PKC & 32,56 & 84,85 & 86,17 & 97,59 & 0,928851 & 94,78 & 99,18 & 0,973369 \\
\hline Phospho & autocatalysis & 13,16 & 96,15 & 79,35 & 93,59 & 0,888392 & 95,96 & 99,15 & 0,97851 \\
\hline Phospho & $\mathrm{CDC} 2$ & 85,71 & 80,9 & 89,55 & 75,95 & 0,916095 & 93,73 & 92,36 & 0,963488 \\
\hline Phosphoserine & - & 97,06 & 91,34 & 94,33 & 95,56 & 0,94975 & 96,87 & 98,02 & 0,912848 \\
\hline Phosphoserine & PKA & 68,42 & 78,31 & 91,67 & 72,64 & 0,922083 & 97,66 & 98,43 & 0,987096 \\
\hline Phosphoserine & PKC & 23,29 & 80,95 & 96,43 & 95,29 & 0,975476 & 94,93 & 98,94 & 0,973939 \\
\hline Phosphoserine & autocatalysis & 10 & 85,71 & 75 & 90,7 & 0,868311 & 91,57 & 99,13 & 0,957295 \\
\hline Phosphoserine & CK2 & 29,73 & 70,97 & 88,14 & 59,77 & 0,882345 & 93,02 & 98,23 & 0,962999 \\
\hline
\end{tabular}


Table 2: Improvement of the performance of the AutoMotifServer 3.0. (Continued)

\begin{tabular}{|c|c|c|c|c|c|c|c|c|c|}
\hline Phosphothreonine & - & 46,12 & 75,46 & 78,16 & 69,73 & 0,828495 & 85,2 & 97,4 & 0,905598 \\
\hline Phosphothreonine & autocatalysis & 0 & 0 & 73,47 & 80 & 0,849347 & 94,96 & 99,82 & 0,974381 \\
\hline Phosphotyrosine & - & 9,32 & 71,2 & 84,24 & 96,69 & 0,91775 & 91,75 & 98,97 & 0,952053 \\
\hline Phosphotyrosine & autocatalysis & 1,41 & 12,5 & 92 & 92 & 0,951404 & 96,82 & 97,21 & 0,979045 \\
\hline Pyrrolidone carboxylic acid & - & 60,31 & 97,11 & 91,58 & 97,92 & 0,955236 & - & - & - \\
\hline Sulfotyrosine & - & 70,19 & 85,88 & 98,39 & 97,6 & 0,988929 & 99,48 & 98,47 & 0,990853 \\
\hline Valine amide & - & 94,29 & 97,06 & 98,57 & 98,57 & 0,986607 & - & - & - \\
\hline Phospho.ELM & - & 98 & 91,68 & - & - & - & 98,56 & 96,07 & 0,949397 \\
\hline Phospho.ELM & Abl & 0 & 0 & - & - & - & 81,82 & 52,94 & 0,862032 \\
\hline Phospho.ELM & AMPK_group & 6,25 & 100 & - & - & - & 100 & 72,22 & 0,975 \\
\hline Phospho.ELM & ATM & 92,98 & 81,54 & - & - & - & 94,87 & 90,24 & 0,967439 \\
\hline Phospho.ELM & CaM-KIlalpha & 41,67 & 88,24 & - & - & - & 100 & 77,42 & 0,981081 \\
\hline Phospho.ELM & $\begin{array}{c}\text { CaM- } \\
\text { KII_group }\end{array}$ & 14,55 & 88,89 & - & - & - & 82,05 & 94,12 & 0,906796 \\
\hline Phospho.ELM & CDK1 & 41,73 & 63,04 & - & - & - & 96,94 & 62,91 & 0,94711 \\
\hline Phospho.ELM & CDK2 & 7,14 & 45,45 & - & - & - & 92,31 & 64,86 & 0,928627 \\
\hline Phospho.ELM & CDK_group & 59,8 & 67,03 & - & - & - & 95,65 & 67,35 & 0,947785 \\
\hline Phospho.ELM & CK1_group & 0 & 0 & - & - & - & 86,67 & 72,22 & 0,911594 \\
\hline Phospho.ELM & CK2 alpha & 38,98 & 67,65 & - & - & - & 78,82 & 70,53 & 0,872036 \\
\hline Phospho.ELM & CK2_group & 43,33 & 72,22 & - & - & - & 73,33 & 64,36 & 0,839759 \\
\hline Phospho.ELM & EGFR & 0 & 0 & - & - & - & 73,17 & 78,95 & 0,852909 \\
\hline Phospho.ELM & Fyn & 0 & 0 & - & - & - & 87,5 & 84,85 & 0,9275 \\
\hline Phospho.ELM & GRK_group & 2,7 & 100 & - & - & - & 83,33 & 90,91 & 0,911404 \\
\hline Phospho.ELM & GSK-3beta & 18,37 & 75 & - & - & - & 85,29 & 64,44 & 0,896282 \\
\hline Phospho.ELM & GSK-3_group & 12,5 & 66,67 & - & - & - & 65,22 & 93,75 & 0,823128 \\
\hline Phospho.ELM & IGF1R & 26,09 & 100 & - & - & - & 85 & 73,91 & 0,90625 \\
\hline Phospho.ELM & IKK_group & 0 & 0 & - & - & - & 95,83 & 92 & 0,973761 \\
\hline Phospho.ELM & InsR & 6,67 & 60 & - & - & - & 70,97 & 88 & 0,848289 \\
\hline Phospho.ELM & Lck & 11,76 & 60 & - & - & - & 80,56 & 74,36 & 0,884596 \\
\hline Phospho.ELM & Lyn & 0 & 0 & - & - & - & 66,67 & 84,62 & 0,825137 \\
\hline Phospho.ELM & MAPK1 & 45,88 & 67,24 & - & - & - & 88,98 & 55,85 & 0,898547 \\
\hline Phospho.ELM & MAPK14 & 4 & 22,22 & - & - & - & 82,35 & 77,78 & 0,896017 \\
\hline Phospho.ELM & MAPK3 & 74,7 & 78,48 & - & - & - & 87,93 & 77,27 & 0,922801 \\
\hline
\end{tabular}


Table 2: Improvement of the performance of the AutoMotifServer 3.0. (Continued)

\begin{tabular}{|c|c|c|c|c|c|c|c|c|c|}
\hline Phospho.ELM & MAPK8 & 14,71 & 41,67 & - & - & - & 91,67 & 84,62 & 0,947523 \\
\hline Phospho.ELM & MAPKAPK2 & 3,03 & 100 & - & - & - & 69,57 & 80 & 0,836332 \\
\hline Phospho.ELM & MAPK_group & 54,9 & 77,78 & - & - & - & 82,35 & 75,68 & 0,894784 \\
\hline Phospho.ELM & PDK-1 & 42,86 & 85,71 & - & - & - & 80 & 95,24 & 0,897283 \\
\hline Phospho.ELM & PKA alpha & 42,42 & 82,35 & - & - & - & 86,96 & 95,24 & 0,931824 \\
\hline Phospho.ELM & PKA_group & 58,15 & 90,43 & - & - & - & 91,74 & 62,99 & 0,904783 \\
\hline Phospho.ELM & PKB_group & 79,76 & 65,05 & - & - & - & 88,14 & 77,61 & 0,924011 \\
\hline Phospho.ELM & PKC alpha & 19,7 & 72,22 & - & - & - & 75,56 & 48,57 & 0,825223 \\
\hline Phospho.ELM & PKC_group & 25,21 & 80 & - & - & - & 82,02 & 84,39 & 0,900038 \\
\hline Phospho.ELM & PLK1 & 0 & 0 & - & - & - & 68,97 & 45,45 & 0,788753 \\
\hline Phospho.ELM & Src & 0,67 & 5 & - & - & - & 74,31 & 65,32 & 0,845308 \\
\hline Phospho.ELM & Syk & 6,67 & 30 & - & - & - & 85,71 & 53,57 & 0,878378 \\
\hline
\end{tabular}

Comparison of best performances of the AMS 2.0 server with the current AMS version 3.0 on best results obtained on the training datasets of respective PTM types. 
Many researchers concentrated their efforts on prediction of four major kinase families, namely CDK, CK2, PKA and PKC. To compare the current technique with the existing ones, we have conducted detailed experiments with those four kinase families from the latest Phospho.ELM dataset. Figure 2 (a-b) shows scopes of AUCs for these four kinase families for the train and test datasets of AMS3. In the current work we have compared the performance of AMS3 with the existing state-of-theart prediction systems for phosphorylation sites in protein sequences. Figure 3 shows a comparative analysis of the current technique with standard predictors, namely GPS, KinasePhos, NetPhosK, PPSP, PredPhospho, Scansite and Meta Predictor. Table 3 lists the comparative performances, i.e. sensitivity, specificity and accuracy, of aforementioned prediction systems (and their variations) with the current one. In another comparison, we have plotted the ROC curves of the four kinase families Figure 4(a-d) for different runs of trainset/testset results for AMS3 and compared the same with claimed ROC values of the standard predictors.

Among the other comparable works in this domain, the NetPhos 2.0 server predicts serine, threonine and tyrosine phosphorylation sites in eukaryotic proteins and NetPhosK 1.0 server [11] predicts kinase specific eukaryotic protein phosphoylation sites. Similar to our approach, both servers use neural network based classifiers for prediction of amino acid sequences. The performance of the NetPhosK server is reported on 5 different PTM types, i.e. PKA, PKC, CaM-II, cdc2 and CKII on independent test datasets. Table 4 shows the comparative analysis of best recognition performances of both the servers on test samples of comparable PTM types. It can be observed that our current technique also shows superior performances in all the cases under consideration. Due to variations in nomenclature and datasets, the further (unbiased) comparison with NetPhosK and other available servers could not be carried out exhaustively.

Average execution time for the current software is around $25 \mathrm{~ms}$ for 100 entries of short amino acid sequences. Each such short sequence contains nine amino acids, extracted from a complete FASTA formatted protein sequence. The experiment is conducted on a moderately powerful desktop with $1.6 \mathrm{GHz}$ processor and $768 \mathrm{MB}$ primary memory in Linux based operating environment.

\section{Discussion}

The present method provides a fast and accurate system for prediction of post-translational modification sites that is capable of classifying highly complex local biological sequence features. The current design of the neural network implements three different network models for each of the PTM types by independently optimizing the net- work weights for optimum recall/precision/AUC values on randomly chosen test patterns.

The efficiency of classification and the prediction power of our method, estimated on training and test datasets for each PTM types using the sensitivity (recall), precision values and area under ROC curves, clearly outperforms the previously reported results. As evident in Tables 2, 3 and 4, the current technique improves the earlier versions of the AutoMotifServer (AMS 1.0, 2.0) and also outperforms the other state-of-the-art systems, GPS, KinasePhos, NetPhosK, PPSP, PredPhospho, Scansite and Meta Predictor, designed for kinase-specific prediction of phosphorylation sites in protein sequences. For comparison with our earlier version of AMS, the new Swiss Prot dataset could not be used because of the new nomenclature in the current version. Therefore, we partially ran the experiments with the old version of the dataset. However in the case of Phospho.ELM, the complete experimentation is done, together with the new version of the Swiss Prot dataset. Significant differences in the performance of differently optimized neural networks (for different PTM types) are observed, yet the AMS 3.0 tool integrates those heterogeneous classification schemes and it is able to boost the precision and recall values independent of a PTM type in comparison. Performances of AMS3 are also evaluated on four kinase families CDK, CK2, PKA and PKC and compared with the aforementioned predictor systems. We could observe that the performance of the current technique is comparable with the best among the rest. However, there are cases when the current technique fails to beat all other predictors. As shown in Figure 3, for the best AUC value for kinase type PKC, Meta Predictor outperforms AMS3. Similarly, in case of kinase type CK2, AMS3 comes third, below NetPhosK and PPSP. In another illustration related to ROC values, similar findings could be observed, where the ROC values of different predictors and their variations mostly come within ROC curves of AMS3 train sets and independent test sets.

There are two key reasons behind the performance boost of the current version of the AMS. Firstly, prudent choice of the feature descriptors from the AAindex database by exhaustive trial and error with different possible features. Secondly, the choice and design of the MLP base classifier. Despite popular choice of SVM in binary classification problems, we have got better accuracy with MLP by exhaustively tuning variety of learning parameters and experimented on a wide range of hidden neuron variations. The prudent choice of train/test ratios in positive and negative samples and the novel idea of optimizing respective networks separately on peak AUC/Recall/Precision values, also boosted the overall performance preserving the generality of the tool. 
Table 3: The best PTM predictors.

\begin{tabular}{|c|c|c|c|c|c|}
\hline & Sensitivity & Specificity & Accuracy & MCC & POS \\
\hline \multicolumn{6}{|l|}{ CDK } \\
\hline GPS & 0,908 & 0,8 & 0,844 & 0,695 & 294 \\
\hline KinasePhos_90 & 0,884 & 0,717 & 0,784 & 0,589 & \\
\hline KinasePhos_95 & 0,799 & 0,837 & 0,822 & 0,632 & \\
\hline KinasePhos_100 & 0,571 & 0,923 & 0,782 & 0,542 & \\
\hline KinasePhos_bitscore & 0,912 & 0,685 & 0,776 & 0,588 & \\
\hline NetPhosK_0.3 & 1 & 0 & 0,4 & $\mathrm{~N} / \mathrm{A}$ & \\
\hline NetPhosK_0.5 & 0,639 & 0,748 & 0,705 & 0,387 & \\
\hline NetPhosK_0.7 & 0,065 & 0,998 & 0,624 & 0,188 & \\
\hline PPSP_highsens & 0,983 & 0,075 & 0,438 & 0,128 & \\
\hline PPSP_balanced & 0,905 & 0,796 & 0,839 & 0,687 & \\
\hline PPSP_highspec & 0,054 & 0,982 & 0,611 & 0,1 & \\
\hline PredPhospho & 0,898 & 0,823 & 0,853 & 0,708 & \\
\hline Scansite_low & 0,667 & 0,884 & 0,797 & 0,571 & \\
\hline Scansite_medium & 0,405 & 0,971 & 0,744 & 0,479 & \\
\hline Scansite_high & 0,153 & 0,993 & 0,657 & 0,29 & \\
\hline Meta Predictor & 0,912 & 0,832 & 0,864 & 0,73 & \\
\hline AMS 3 (trainset) & 0,957 & 0,94 & 0,941 & & 104 \\
\hline AMS 3 (testset) & 0,971 & 0,94 & 0,943 & & \\
\hline CK2 & & & & & 229 \\
\hline GPS & 0,699 & 0,895 & 0,816 & 0,613 & \\
\hline KinasePhos_90 & 0,581 & 0,904 & 0,774 & 0,523 & \\
\hline KinasePhos_95 & 0,476 & 0,95 & 0,76 & 0,504 & \\
\hline KinasePhos_100 & 0,266 & 0,985 & 0,698 & 0,386 & \\
\hline KinasePhos_bitscore & 0,594 & 0,901 & 0,778 & 0,53 & \\
\hline NetPhosK_0.3 & 0,961 & 0,525 & 0,699 & 0,506 & \\
\hline NetPhosK_0.5 & 0,755 & 0,948 & 0,871 & 0,73 & \\
\hline NetPhosK_0.7 & 0,245 & 1 & 0,698 & 0,403 & \\
\hline PPSP_highsens & 0,93 & 0,227 & 0,509 & 0,208 & \\
\hline PPSP_balanced & 0,742 & 0,933 & 0,857 & 0,7 & \\
\hline PPSP_highspec & 0,048 & 1 & 0,619 & 0,171 & \\
\hline PredPhospho & 0,594 & 0,959 & 0,813 & 0,616 & \\
\hline Scansite_low & 0,576 & 0,983 & 0,82 & 0,64 & \\
\hline Scansite_medium & 0,38 & 0,997 & 0,75 & 0,512 & \\
\hline Scansite_high & 0,135 & 1 & 0,654 & 0,293 & \\
\hline Meta Predictor & 0,878 & 0,904 & 0,893 & 0,779 & \\
\hline AMS 3 (trainset) & 0,733 & 0,95 & 0,921 & & 248 \\
\hline AMS 3 (testset) & 0,675 & 0,94 & 0,921 & & \\
\hline \multicolumn{6}{|l|}{ PKA } \\
\hline GPS & 0,817 & 0,809 & 0,812 & 0,618 & 360 \\
\hline KinasePhos_90 & 0,722 & 0,843 & 0,794 & 0,569 & \\
\hline KinasePhos_95 & 0,65 & 0,887 & 0,792 & 0,56 & \\
\hline KinasePhos_100 & 0,361 & 0,952 & 0,716 & 0,405 & \\
\hline
\end{tabular}


Table 3: The best PTM predictors. (Continued)

\begin{tabular}{|c|c|c|c|c|c|}
\hline KinasePhos_bitscore & 0,775 & 0,804 & 0,792 & 0,573 & \\
\hline NetPhosK_0.3 & 0,878 & 0,724 & 0,786 & 0,59 & \\
\hline NetPhosK_0.5 & 0,694 & 0,874 & 0,802 & 0,583 & \\
\hline NetPhosK_0.7 & 0,483 & 0,959 & 0,769 & 0,525 & \\
\hline PPSP_highsens & 0,967 & 0,231 & 0,526 & 0,27 & \\
\hline PPSP_balanced & 0,85 & 0,806 & 0,823 & 0,645 & \\
\hline PPSP_highspec & 0,008 & 0,998 & 0,602 & 0,048 & \\
\hline PredPhospho & 0,808 & 0,839 & 0,827 & 0,642 & \\
\hline Scansite_low & 0,644 & 0,917 & 0,808 & 0,596 & \\
\hline Scansite_medium & 0,422 & 0,981 & 0,758 & 0,515 & \\
\hline Scansite_high & 0,158 & 0,991 & 0,658 & 0,288 & \\
\hline Meta Predictor & 0,883 & 0,828 & 0,85 & 0,699 & \\
\hline AMS 3 (trainset) & 0,917 & 0,891 & 0,896 & & 345 \\
\hline AMS 3 (testset) & 0,87 & 0,892 & 0,89 & & \\
\hline \multicolumn{6}{|l|}{ PKC } \\
\hline GPS & 0,718 & 0,753 & 0,739 & 0,466 & 348 \\
\hline KinasePhos_90 & 0,649 & 0,789 & 0,733 & 0,441 & \\
\hline KinasePhos_95 & 0,48 & 0,864 & 0,71 & 0,378 & \\
\hline KinasePhos_100 & 0,129 & 0,977 & 0,638 & 0,211 & \\
\hline KinasePhos_bitscore & 0,687 & 0,722 & 0,708 & 0,404 & \\
\hline NetPhosK_0.3 & 0,716 & 0,695 & 0,703 & 0,403 & \\
\hline NetPhosK_0.5 & 0,491 & 0,841 & 0,701 & 0,358 & \\
\hline NetPhosK_0.7 & 0,333 & 0,935 & 0,694 & 0,348 & \\
\hline PPSP_highsens & 0,954 & 0,274 & 0,546 & 0,289 & \\
\hline PPSP_balanced & 0,741 & 0,743 & 0,743 & 0,477 & \\
\hline PPSP_highspec & 0,006 & 1 & 0,602 & 0,059 & \\
\hline PredPhospho & 0,598 & 0,805 & 0,722 & 0,412 & \\
\hline Scansite_low & 0,411 & 0,866 & 0,684 & 0,315 & \\
\hline Scansite_medium & 0,17 & 0,946 & 0,636 & 0,189 & \\
\hline Scansite_high & 0,069 & 0,994 & 0,624 & 0,179 & \\
\hline Meta Predictor & 0,773 & 0,791 & 0,784 & 0,558 & \\
\hline AMS 3 (trainset) & 0,82 & 0,98 & 0,961 & & 267 \\
\hline AMS 3 (testset) & 0,629 & 0,931 & 0,912 & & \\
\hline
\end{tabular}

The current technique is very fast in comparison to our previously developed SVM based version of the server. As mentioned earlier, the average prediction time for 100 short amino acid sequences is estimated at around $25 \mathrm{~ms}$ on a standard desktop computer. In a nutshell, the MLP based pattern classifier, with independent recall/precision/AUC optimized networks, along with an effective feature descriptor is found to be more suitable to the massive prediction of post-translational modifications for whole proteomes. The availability of the precompiled, standalone version allows for high-throughput screening of large sequence datasets, the main problem that the scientific proteome community is now considering heavily.

\section{Conclusions}

Summarizing, the AMS 3.0 tool integrates heterogeneous classification schemes for different PTM types, and it is designed to boost both the efficiency and speed in comparison with previously presented computational methods. Our fast and accurate system for prediction of post- 


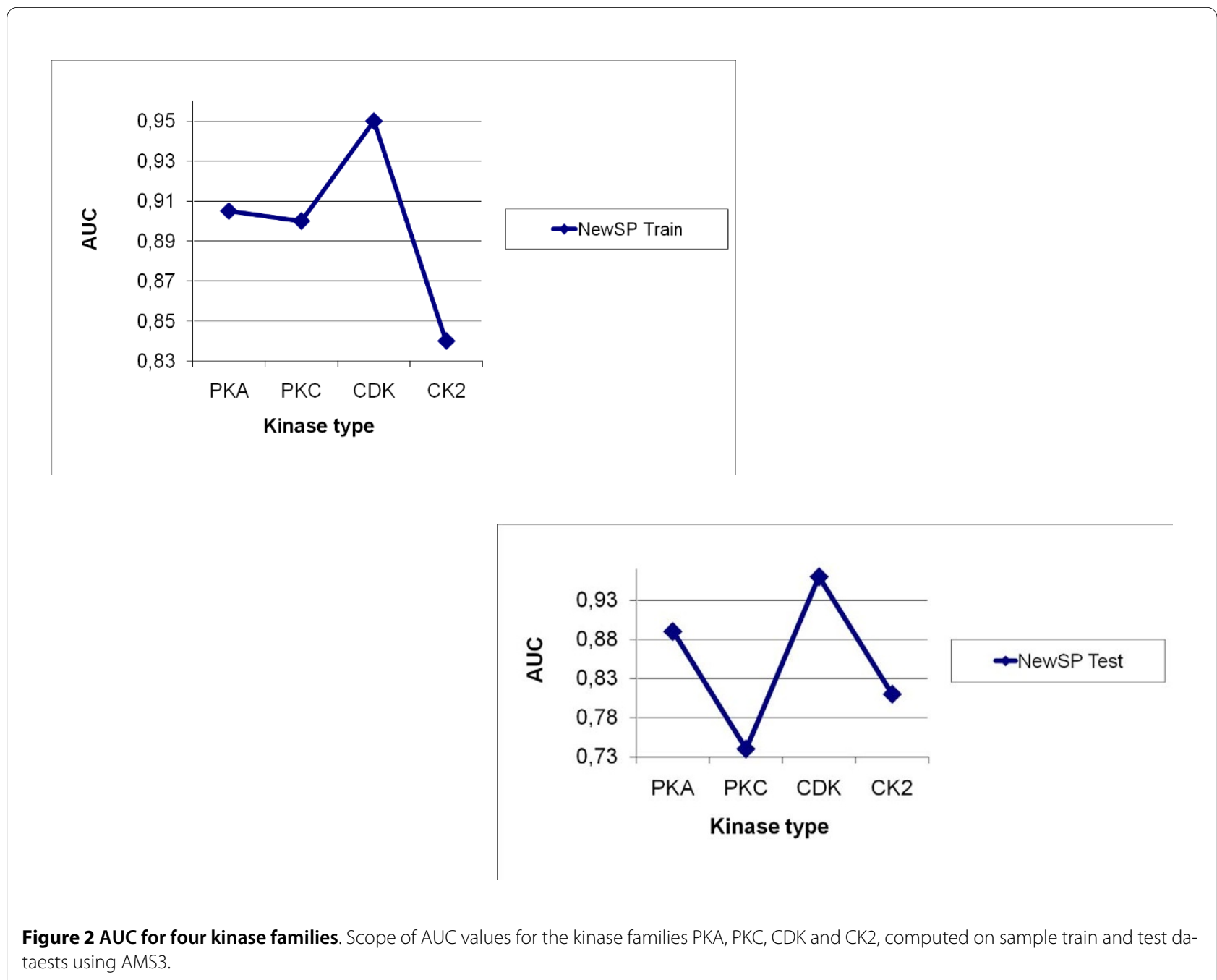

translational modification sites in protein sequences is capable of classifying highly complex and nonlinear biological sequence patterns. We have implemented three different network models for each of the PTM types by optimizing the model for optimum recall/precision/AUC. The features for the current experiment are chosen by exhaustive experimentation on the AAindex database, based on the previously proven amino acid characteristics for prediction of side chain interaction sites, secondary structure information and related attributes. The developed Predictor tool, reads primary protein sequences in FASTA format and decides whether an overlapping short amino acid sequence qualifies for a potential PTM site or not, along with a probabilistic confidence for such a decision. The user also specifies the type of PTM for a specific prediction and the nature of optimization required (based on AUC area, Recall and Precision values).

The training datasets and precompiled binaries for AMS 3.0 tool are available at http://bio.icm.edu.pl/ dar- $\underline{\text { man/ams } 3}$ and the source code at http:// code.google.com/p/automotifserver under the Apache 2.0 license scheme.

\section{Methods}

Current dataset is extracted from the Swiss Prot Release 57.5 (dated 07-Jul-2009, consisting from 470,369 entries). All these data samples are available for free download from http://www.uniprot.org/. The dataset consists of 9 amino acid long sequence fragments centered on the post-translationally modified site used as positive instances. The negative instances were randomly selected such that they do not include experimentally verified PTM sites of any type. For each of the known thirty four (34) PTM types under consideration, separate positive and negative datasets were collected. We have also used Phospho.ELM dataset version 8.2 downloaded from http://phospho.elm.eu.org/dataset.html web site (April 2009). Phospho.ELM version 8.2 contains 4687 substrate proteins covering 2217 tyrosine, 14518 serine and 2914 


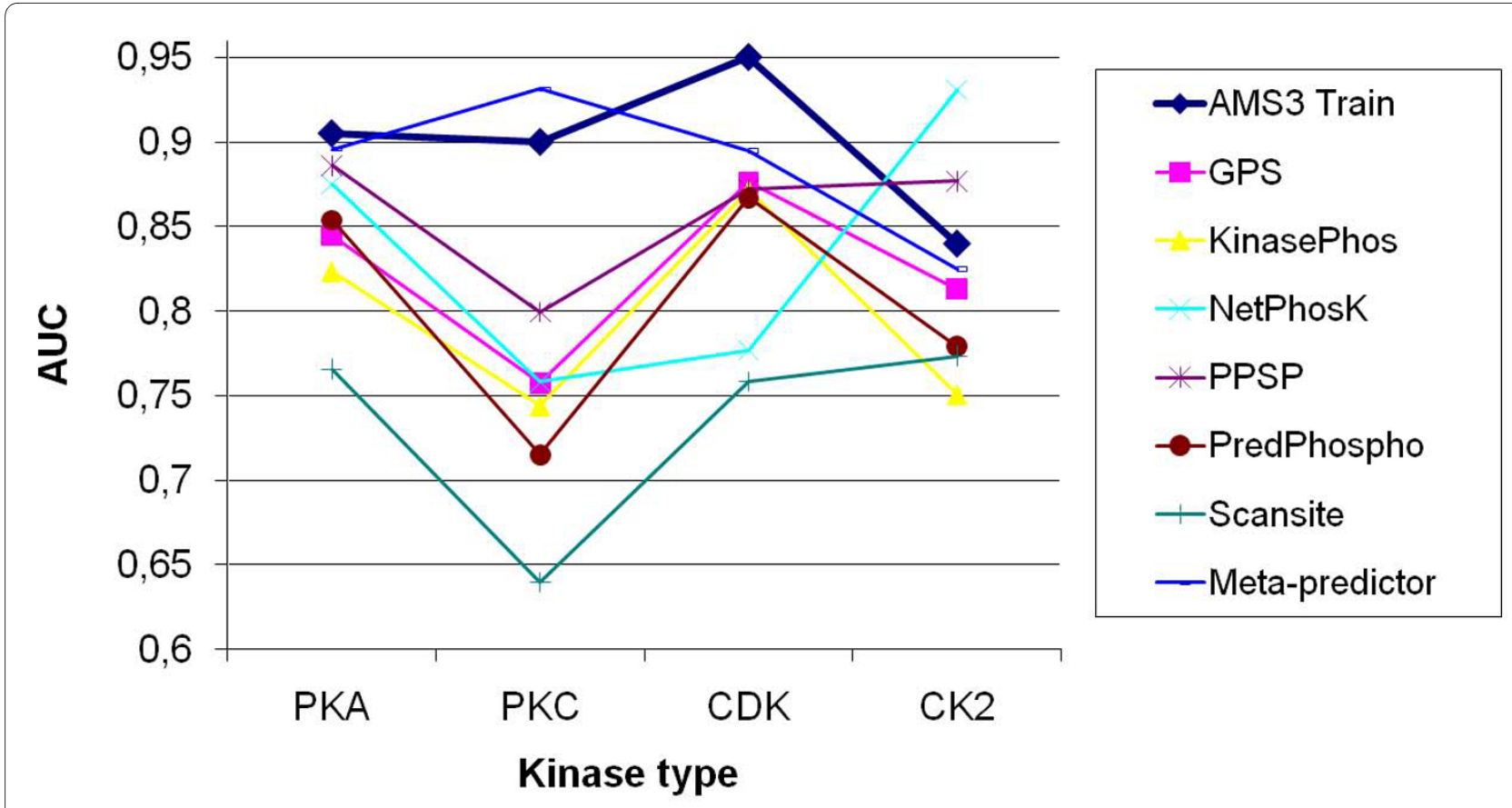

Figure 3 AUC values for predictors. Comparison of scope of AUC best values for the kinase families PKA, PKC, CDK and CK2, using AMS3, GPS, KinasePhos, NetPhosK, PPSP, PredPhospho, Scansite and Meta Predictor.

threonine instances. Data from high-throughput experiments have been included.

The features for the current experiment are chosen by exhaustive optimization and search in all different hundreds (exactly 544 in the current version) of AAindex database release $9.0 \mathrm{http}: / / \mathrm{www}$.genome.jp/aaindex/. Different features are considered based on their previously proven characteristics for the prediction of side chain interaction sites and secondary structure information. More specifically, features based on different side-chain interaction parameters like side-chain volume/hydrophobicity values; some typical amino acid attributes like hydration number, transfer free energy; information value for accessibility, surface tension, molecular weight etc.; and different secondary structure prediction parameters are considered for the selection of the optimum feature subset used here. We initially chose 15 different amino acid features (as shown in Table 1) from the AAindex database. Different combinations of those features descriptors are heuristically evaluated on sample train/ test datasets of different representative PTM types (Phospho-PKA, Phospho-PKC, Phospho-autocatalysis and Phospho-CDC2). After exhaustive trial and error, ten different features are found that generate optimal recognition performance on the test datasets under consideration. Table 1 shows the list of accepted/rejected feature descriptors considered in the procedure. Possible reasons behind rejection of some apparently significant features (like ARGP820101, WARP780101 etc.) may be redundancy, or high correlation between the feature values. In other word, the rejected features failed to provide complementary/additional information to the existing feature set. The final list of accepted feature descriptors constitute a 90 dimensional feature vector for evaluation of recognition performances using an MLP based classifier on the benchmarking datasets for different PTM types under consideration. The chosen set of features can therefore be used as the best proven attributes related to post transactional modifications in amino acid sequences. Table 1 briefly lists these features (and the rejected ones) along with their AAindex accession numbers and short references.

A feed-forward artificial neural network is trained with back-propagation learning algorithm to optimize the classification accuracy between the positive and the negative samples in the randomly chosen test dataset. The optimization procedure is tuned to produce separately optimum recall, precision and the AUC area for the test dataset chosen for each of the PTM types. This is required to address specific requirements from the biologists, generating high recall/precision values for any given query sequence, using respective recall/precision optimized network setups. Also, the network setup for optimum AUC area gives balanced prediction for query sequence, resulting in moderately high both recall and precision values. The performance of each model is 


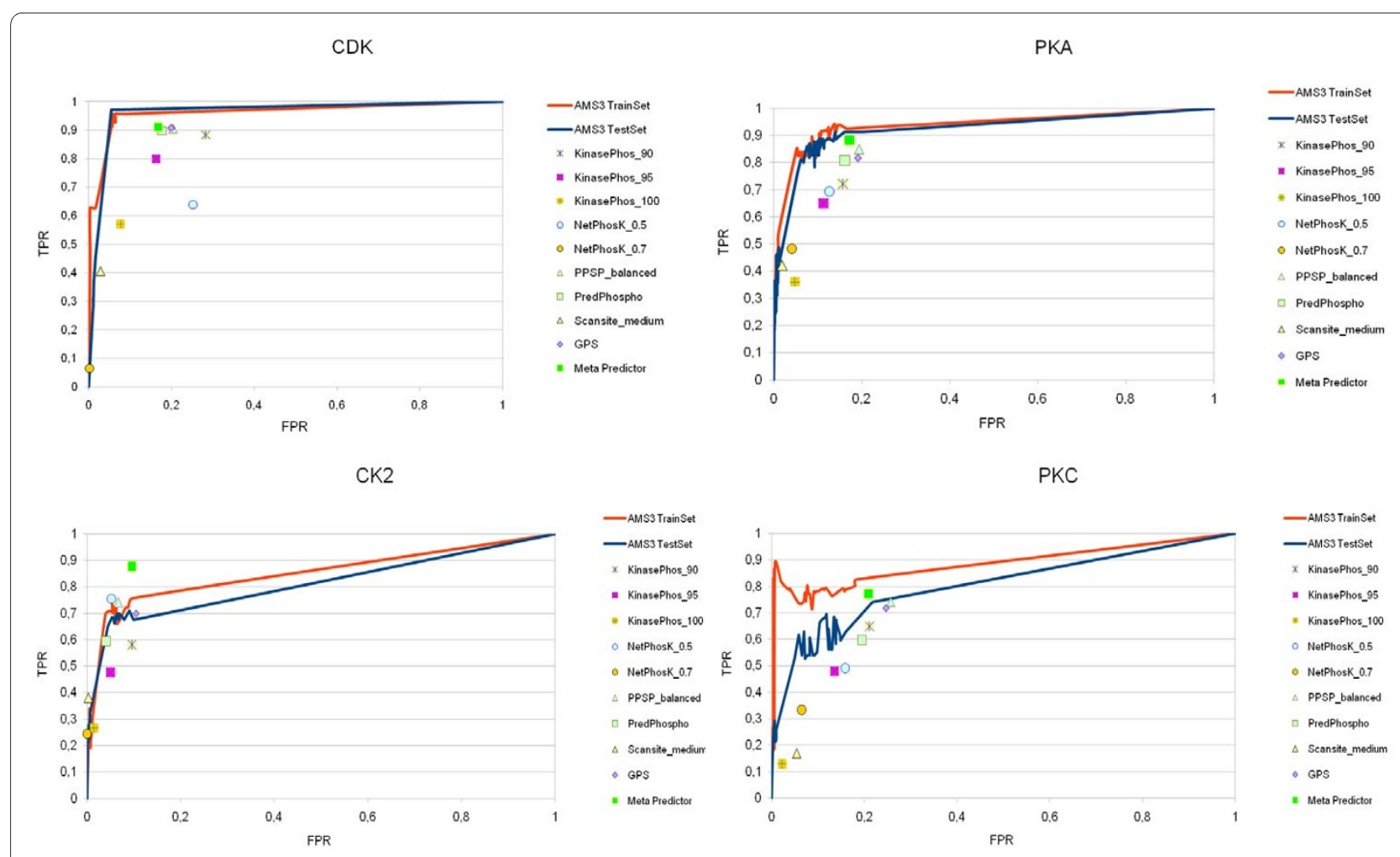

Figure 4 ROC values for four kinase families. Comparison of ROC values for the kinase families PKA, PKC, CDK and CK2, using GPS, KinasePhos, NetPhosK, PPSP, PredPhospho, Scansite and Meta Predictor with the corresponding ROC curves for training and test datasets using AMS3.

therefore calculated for both the training and the test datasets under consideration in three different optimization models. The classification results are generated along with a probabilistic confidence measure for such decision. More specifically, the designed neural network model generates a confidence measure $C_{i j}\left(0 \leq C_{i j} \leq 1\right)$ for the $i^{\text {th }}$ query sequence in the $j^{\text {th }}$ class (in this experiment, either positive or negative). This confidence measure is estimated as the normalized responses $\left(R_{j}\right)$ of respective output neurons in the output layer of the MLP. Such that, for an $i^{t h}$ sequence:

$$
C_{i j}=\frac{R_{j}}{\sum_{k} R_{k}}
$$

An MLP consists of one input layer, one output layer and one (or more) hidden or intermediate layer(s), as shown in Figure 1. The output from every neuron in a layer of the MLP is connected to all inputs of each neuron in the immediate next layer of the same as also illustrated in Figure 1. Neurons in the input layer of the MLP are dummy neurons, as they are used simply to pass on the

Table 4: NetPhosK and AMS web servers.

\begin{tabular}{lcccc}
\hline & Positives & NetPhosK & & \multicolumn{2}{c}{ AMS 3.0 } \\
& & Recall & Positives & 87.5 \\
\hline PKA & 258 & 82 & 121 & 70.83 \\
PKC & 193 & 62 & 118 & 82.05 \\
CaM-II & 26 & 73 & 57 & 88.24 \\
cdc2 & 22 & 37 & 84 & 248 \\
CKII & 85 & 75 & 23.3 \\
\hline
\end{tabular}

Comparison of best recognition performances on independent test sets between the servers NetPhosK and AMS 3.0. 
input to the next layer just by computing an identity function.

The numbers of neurons in the input and the output layers of an MLP are chosen as respectively ninety and two for the current problem, in order to match the dimensionality of the input feature descriptor vector and the number of output classes (positives and negatives) respectively. The number of neurons in other layers and the number of layers in the MLP are determined by exhaustive trial and error method during its training. The MLP used for the present work requires supervised training. During training of an MLP weights or strengths of neuron-to-neuron connections, also called synapses, are iteratively tuned so that the MLP can respond appropriately to all training data and also to other data, not considered at the time of training. Learning and generalization abilities of an ANN are determined on the basis of how best it can respond under these two respective situations.

The MLP classifier designed for the present work is trained with the Back Propagation (BP) algorithm. The algorithm is applied to minimize the sum of the squared errors for the training samples by conducting a gradient descent search in the weight space. The number of neurons in a hidden layer is also varied during its training.

In our work we have implemented random sub-sampling validation, to estimate the unbiased error rate of the designed technique. This method randomly splits the dataset into training and test (validation) data. For each such split, the classifier learns to the training data, and predictive accuracy is assessed using the test data. The results are then averaged over multiple such splits. For most of the PTM types, the training and test samples for positive instances are populated in the ratio of 4:1 from all available positive samples. The number of negative samples for each type of PTM is chosen to be significantly larger than the positive samples for both the train and test datasets (the ratio of positive, negative samples is maintained as 1:5 in most cases). Random sub-sampling produces better error estimates than a single train-and-test split. The advantage of this method (over $k$-fold cross validation) is that the proportion of the training/validation split is not dependent on the number of iterations (folds). In the current work we have performed three random splits in the positive/negative datasets for each PTM. This is done so to eliminate possible bias during the training procedure in any given train/test dataset combination. The neural network for each PTM type is trained separately for the best possible AUC, the recall and precision values on the three randomly chosen independent test datasets. Each of these experiments, for any PTM type, is repeated at least 10 times by varying number of neurons in the hidden layer of the network. More specifically, the experiment is conducted with variation of the number of hidden neurons starting from 2 up to 20 in steps of 2. The optimum networks, giving high AUC, recall and precision values with low error rates, are saved for further consideration.

The problem of overfitting is addressed by optimizing each training network on independent test datasets, i.e., during the iterative training process the network weights that maximizes recognition accuracy on a separate test set (not the training set) is saved. Three random runs of training and test sample sets are considered for generating AUC, Sensitivity (Recall) and Precision optimized neural network models for design of the software tool. Average training and test set accuracies over these three and average over AUC and Recall accuracies are given in the in the Additional file 2.

Leave-one-out cross-validation (LOOCV) often works well for estimating generalization error for continuous error functions such as the mean squared error, but it may perform poorly for discontinuous error functions such as the number of misclassified cases (like the cases considered here). LOOCV can also run into trouble with various model-selection methods. Again, one problem is lack of continuity - a small change in the data can cause a large change in the model selected [32]. LOOCV is usually very expensive from computational point of view because of the large number of times the training process is repeated. Due to the large training data sizes for many PTM types and problem of choosing one single training model for each PTM type, LOOCV methodology is adopted in our experiments for only part of PTM types.

The developed software tool executes in two phases. In the first part, a Sequence Generator program reads any number of protein sequences in FASTA format, written in an input file and generates 9 amino acid long overlapping sequences for prediction. The Predictor program reads these short sequences and generates output. The user can specify the type of PTM for specific prediction and the nature of optimization required (based on AUC area, Recall or Precision values). The output is generated in the following format both as a binary decision (whether the sequence qualifies for a potential PTM site or not) and as a probabilistic confidence measure $\left(C_{i j}\right.$, for the $i^{\text {th }}$ query sequence in the decision class) for each short amino acid sequence, given as an input to the Predictor program. For example:

$\begin{array}{lll}\text { LCLYTHIGR } & 0 & 0.9853 \\ \text { CLYTHIGRN } & 0 & 0.9855 \\ \text { LYTHIGRNI } & 1 & 0.7823 \\ \text { YTHIGRNIY } & 0 & 0.9667 \\ \text { THIGRNIYY } & 0 & 0.9132 \\ \text { HIGRNIYYG } & 0 & 0.9763,\end{array}$

where, 0 and 1 signify potential negative and positive sequences respectively. 


\section{Additional material}

Additional file 1 Supplementary materials for the publication. Supplementary.xIs contains detailed results of training and testing of AMS 3.0 tool in MS Excel file format.

\section{Additional file 2 PTM predictor software with training data}

PTM_Archive.tar.gz includes the predictor software binaries together with all trained ANNs in tar.gz compressed form.

\section{Authors' contributions}

Both authors read and approved the final version of the manuscript. SB was responsible for training of neural network, writing the MLP code, performing additional tests. DMP was involved in preparing the training dataset, designing the AMS 3.0 meta-code and its algorithm. Both authors contributed equally to the writing of manuscript.

\section{Acknowledgements}

This work was supported by EC BioSapiens (LHSG-CT-2003-503265), and OxyGreen (KBBE-2007-212281) 6FP projects, as well as the Polish Ministry of Education and Science (N301 159735, N518 409238, PBZ-MNil-2/1/2005 and others). Authors are also indebted to the contributions of the Centre for Microprocessor Applications for Training Education and Research (CMATER), Computer Science and Engineering Department, Jadavpur University, India, for providing necessary infrastructural facilities during the progress of the work. Authors also acknowledge the contributions of many students, researchers and colleagues of CMATER in developing several key modules of the training and prediction routines, now made available in public domain. The authors would like to thank Marcin Kierczak, PhD for his significant help with the automatic processing of the Swiss-Prot database, Prof. Marek Niezgódka, Dr Anna Trykozko and Krzysztof Ginalski, PhD for their support of the scientific visit of SB at ICM, University of Warsaw.

\section{Author Details}

'Department of Computer Science and Engineering, Jadavpur University, Kolkata - 700032, India and 2Interdisciplinary Centre for Mathematical and Computational Modeling, University of Warsaw, 5a Street, 02-106 Warsaw, Poland

Received: 1 December 2009 Accepted: 28 April 2010

Published: 28 April 2010

\section{References}

1. Bairoch A, Apweiler R: The SWISS-PROT protein sequence data bank and its supplement TrEMBL in 1999. Nucleic acids research 1999, 27(1):49-54.

2. Diella F, Cameron S, Gemund C, Linding R, Via A, Kuster B, Sicheritz-Ponten T, Blom N, Gibson TJ: Phospho.ELM: a database of experimentally verified phosphorylation sites in eukaryotic proteins. $B M C$ bioinformatics 2004, 5:79.

3. Diella F, Gould CM, Chica C, Via A, Gibson TJ: Phospho.ELM: a database of phosphorylation sites--update 2008. Nucleic acids research 2008:D240-244

4. Puntervoll P, Linding R, Gemund C, Chabanis-Davidson S, Mattingsdal M, Cameron S, Martin DM, Ausiello G, Brannetti B, Costantini A, et al.: ELM server: A new resource for investigating short functional sites in modular eukaryotic proteins. Nucleic acids research 2003, 31(13):3625-3630

5. Sigrist CJ, Cerutti L, Hulo N, Gattiker A, Falquet L, Pagni M, Bairoch A, Bucher P: PROSITE: a documented database using patterns and profiles as motif descriptors. Briefings in bioinformatics 2002, 3(3):265-274.

6. Yaffe MB, Leparc GG, Lai J, Obata T, Volinia S, Cantley LC: A motif-based profile scanning approach for genome-wide prediction of signaling pathways. Nat Biotechnol 2001, 19(4):348-353.

7. Xue Y, Zhou F, Zhu M, Ahmed K, Chen G, Yao X: GPS: a comprehensive www server for phosphorylation sites prediction. Nucleic acids research 2005:W184-187.

8. Xue $Y$, Li A, Wang L, Feng H, Yao X: PPSP: prediction of PK-specific phosphorylation site with Bayesian decision theory. BMC bioinformatics 2006, 7:163

9. Koenig M, Grabe N: Highly specific prediction of phosphorylation sites in proteins. Bioinformatics (Oxford, England) 2004, 20(18):3620-3627.
10. Blom N, Gammeltoft S, Brunak S: Sequence and structure-based prediction of eukaryotic protein phosphorylation sites. J Mol Biol 1999, 294(5):1351-1362.

11. Blom N, Sicheritz-Ponten T, Gupta R, Gammeltoft S, Brunak S: Prediction of post-translational glycosylation and phosphorylation of proteins from the amino acid sequence. Proteomics 2004, 4(6):1633-1649.

12. Hjerrild M, Stensballe A, Rasmussen TE, Kofoed CB, Blom N, Sicheritz Ponten T, Larsen MR, Brunak S, Jensen ON, Gammeltoft S: Identification of phosphorylation sites in protein kinase A substrates using artificial neural networks and mass spectrometry. J Proteome Res 2004, 3(3):426-433.

13. Julenius K, Molgaard A, Gupta R, Brunak S: Prediction, conservation analysis, and structural characterization of mammalian mucin-type Oglycosylation sites. Glycobiology 2005, 15(2):153-164.

14. Gupta R, Jung E, Gooley AA, Williams KL, Brunak S, Hansen J: Scanning the available Dictyostelium discoideum proteome for O-linked GlcNAc glycosylation sites using neural networks. Glycobiology 1999, 9(10):1009-1022.

15. Gupta R, Brunak S: Prediction of glycosylation across the human proteome and the correlation to protein function. Pac Symp Biocomput 2002:310-322

16. Blom N, Hansen J, Blaas D, Brunak S: Cleavage site analysis in picornaviral polyproteins: discovering cellular targets by neural networks. Protein Sci 1996, 5(11):2203-2216.

17. Duckert $P$, Brunak S, Blom N: Prediction of proprotein convertase cleavage sites. Protein Eng Des Sel 2004, 17(1):107-112.

18. Kiemer L, Lund O, Brunak S, Blom N: Coronavirus 3CLpro proteinase cleavage sites: possible relevance to SARS virus pathology. BMC bioinformatics 2004, 5:72.

19. Bologna G, Yvon C, Duvaud S, Veuthey AL: N-Terminal myristoylation predictions by ensembles of neural networks. Proteomics 2004, 4(6):1626-1632

20. Shao J, Xu D, Tsai SN, Wang Y, Ngai SM: Computational identification of protein methylation sites through bi-profile Bayes feature extraction. PLoS One 2009, 4(3): e4920

21. Li S, Li H, Li M, Shyr Y, Xie L, Li Y: Improved prediction of lysine acetylation by support vector machines. Protein Pept Lett 2009, 16(8):977-983.

22. Kim JH, Lee J, Oh B, Kimm K, Koh I: Prediction of phosphorylation sites using SVMs. Bioinformatics (Oxford, England) 2004, 20(17):3179-3184.

23. Plewczynski D, Tkacz A, Wyrwicz LS, Rychlewski L: AutoMotif server: prediction of single residue post-translational modifications in proteins. Bioinformatics (Oxford, England) 2005, 21(10):2525-2527.

24. Wong $\mathrm{YH}$, Lee TY, Liang HK, Huang $\mathrm{CM}$, Wang TY, Yang YH, Chu CH, Huang HD, Ko MT, Hwang JK: KinasePhos 2.0: a web server for identifying protein kinase-specific phosphorylation sites based on sequences and coupling patterns. Nucleic acids research 2007:W588-594.

25. Monigatti F, Gasteiger E, Bairoch A, Jung E: The Sulfinator: predicting tyrosine sulfation sites in protein sequences. Bioinformatics (Oxford, England) 2002, 18(5):769-770.

26. Hamby SE, Hirst JD: Prediction of glycosylation sites using random forests. BMC bioinformatics 2008, 9:500.

27. Wan J, Kang S, Tang C, Yan J, Ren Y, Liu J, Gao X, Banerjee A, Ellis LB, Li T: Meta-prediction of phosphorylation sites with weighted voting and restricted grid search parameter selection. Nucleic acids research 2008 , 36(4):e22.

28. Lee $T Y$, Huang HD, Hung JH, Huang HY, Yang YS, Wang TH: dbPTM: an information repository of protein post-translational modification. Nucleic acids research 2006:D622-627.

29. Miller ML, Blom N: Kinase-specific prediction of protein phosphorylation sites. Methods Mol Biol 2009, 527:299-310.

30. Plewczynski D, Tkacz A, Wyrwicz LS, Rychlewski L, Ginalski K: AutoMotif Server for prediction of phosphorylation sites in proteins using support vector machine: 2007 update. J Mol Model 2008, 14(1):69-76.

31. Fawcett T: An introduction to ROC analysis. Pattern Recognition Letters 2006, 27:861-874

32. Breiman L: Heuristics of instability and stabilization in model selection. Annals of Statistics 1996, 24:2350-2383.

doi: $10.1186 / 1471-2105-11-210$

Cite this article as: Basu and Plewczynski, AMS 3.0: prediction of post-translational modifications BMC Bioinformatics 2010, 11:210 